\title{
An adult case with LACHT syndrome (Mardini-Nyhan association)
}

Mohammad esmaeil zanganehfar ${ }^{1}$, Hamidreza Pouraliakbar ${ }^{2}$, Maryam Shojaeifard $^{2}$, and MohammadFaryad Mahmoudi ${ }^{2}$

${ }^{1}$ Affiliation not available

${ }^{2}$ Rajaie Cardiovascular Medical and Research Center

September 25, 2021

\begin{abstract}
Mardini-Nyhan or LACHT (lung anomaly, congenital heart defect, and thumb anomaly) syndrome is extremely rare. The literature contains only 12 reported cases in the pediatric population. We herein describe an adult patient with this syndrome.
\end{abstract}

\section{An adult case with LACHT syndrome (Mardini-Nyhan association)}

Mohammad Esmail Zanganehfar ${ }^{1}$, MD; Hamid Reza Pouraliakbar ${ }^{1}$, MD; Maryam Shojaeifard ${ }^{2}$, MD; Mohammad Faryad Mahmoudi ${ }^{1}$, MD

${ }^{1}$ Rajaie Cardiovascular Medical and Research Center, Iran University of Medical Sciences, Tehran, Iran

${ }^{2}$ Echocardiography Research Center, Rajaie Cardiovascular Medical and Research Center, Iran University of Medical Sciences, Tehran, Iran

Corresponding author:

Dr Mohammad Esmail Zanganehfar

Email: zanganehfar97@gmail.com

Address: Rajaie Cardiovascular Medical and Research Center, Iran University of Medical Sciences, Vali-Asr Ave, Tehran 1995614331, Iran.

Phone number: +989124190278

\section{ABSTRACT}

Mardini-Nyhan or LACHT (lung anomaly, congenital heart defect, and thumb anomaly) syndrome is extremely rare. The literature contains only 12 reported cases in the pediatric population. We herein describe an adult patient with this syndrome.

A 52-year-old woman was referred to our center for the closure of an atrial septal defect (ASD), which had been discovered during evaluation for chronic mild dyspnea and an abnormal electrocardiogram (ECG).

The patient had no prominent past medical history. Poor R-wave progression with incomplete right bundle branch block in ECG prompted further evaluation for associated cardiac anomalies and the possibility of ASD device closure.

Transesophageal echocardiography showed a moderate-sized ASD (Panel A), mild right ventricular enlargement, increased systolic pulmonary artery pressure ([?] $50 \mathrm{~mm} \mathrm{Hg}$ ), and normal right pulmonary venous drainage; however, the left pulmonary veins were not visible. 
Chest X-ray unexpectedly showed total agenesis of the left lung, increased cardiothoracic ratio, the shifting of the heart and the mediastinum to the left side, and the upward displacement of the left diaphragm (Panel B).

Cardiac computed tomography confirmed the chest X-ray findings by illustrating agenesis of the left main bronchus from the proximal part, complete agenesis of the left lung (Panel C), a large secundum ASD (Panel D), dilatation of the main pulmonary artery (red arrow) and the right main pulmonary artery (yellow arrow), and absence of the left main pulmonary artery (Panel E \& Panel F).

Heart catheterization for an accurate evaluation of pulmonary hypertension demonstrated mild hypertension (pulmonary artery pressure $=55 \mathrm{~mm} \mathrm{Hg}$ ). Pulmonary artery injection showed dilated main and right pulmonary arteries without a visible left-sided branch (Panel G \& Panel I). Further evaluation revealed congenital left thumb hypoplasia. Hand X-ray illustrated a hypoplastic left first metacarpal (Panel H).

All the mentioned findings indicated a syndromic manifestation, termed "lung anomaly, congenital heart defect, and thumb anomaly (LACHT)" or Mardini-Nyhan syndrome. ${ }^{1}$

\section{REFERENCES}

Hastings R, Harding D, Donaldson A, et al. Mardini-Nyhan association (lung agenesis, congenital heart, and thumb anomalies): three new cases and possible recurrence in a sib-is there a distinct recessive syndrome? Am J Med Genet A. 2009;149A(12):2838-2842.

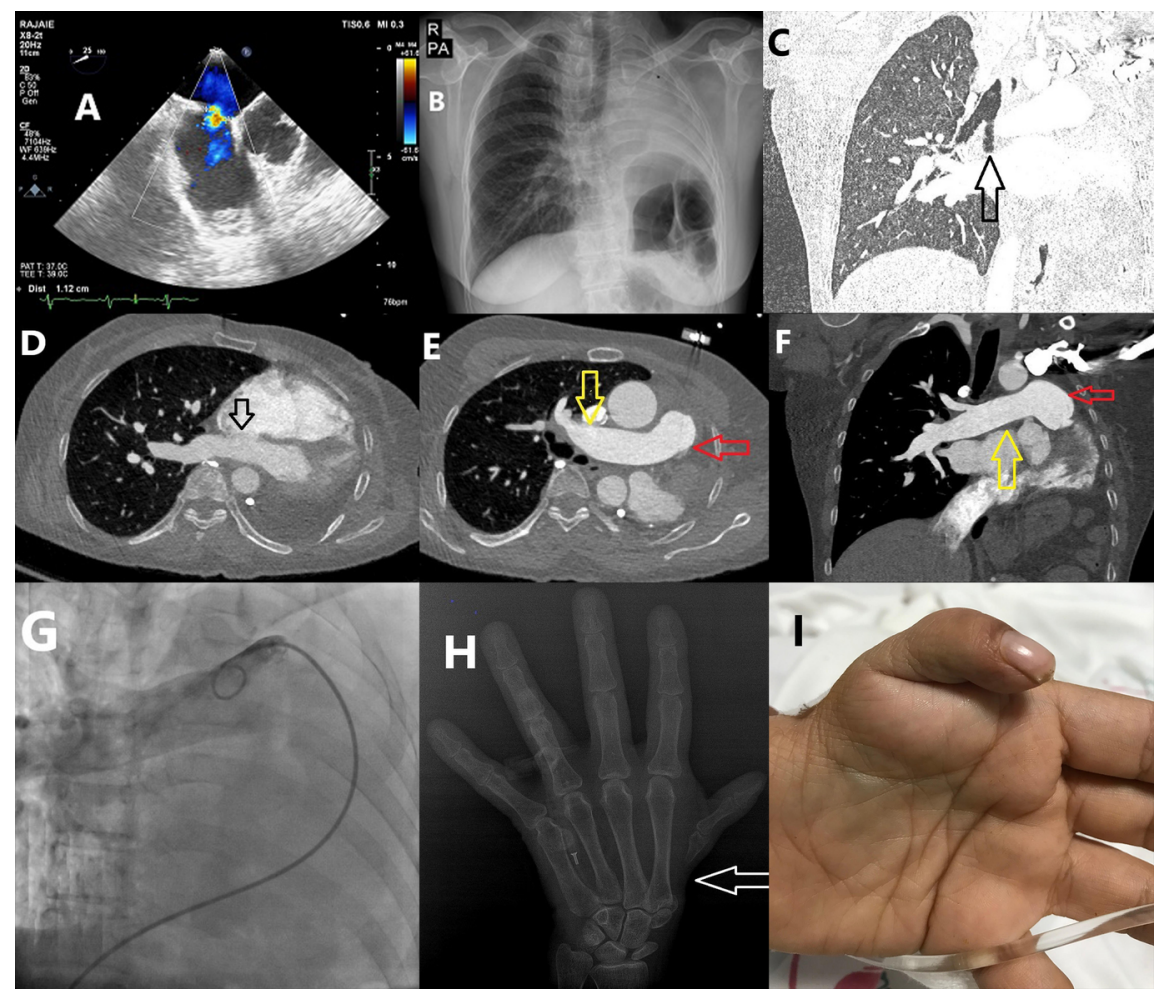

